\title{
The VEINES-QOL/Sym questionnaire is a reliable and valid disease-specific quality of life measure for deep vein thrombosis in elderly patients
}

\author{
M. Méan • A. Limacher • S. R. Kahn • \\ D. Aujesky
}

Accepted: 18 April 2014/Published online: 4 May 2014

(c) Springer International Publishing Switzerland 2014

\begin{abstract}
Purpose To prospectively evaluate the psychometric properties of the Venous Insufficiency Epidemiological and Economic Study (VEINES-QOL/Sym) questionnaire, an instrument to measure disease-specific quality of life and symptoms in elderly patients with deep vein thrombosis (DVT), and to validate a German version of the questionnaire. Methods In a prospective multicenter cohort study of patients aged $\geq 65$ years with acute venous thromboembolism, we used standard psychometric tests and criteria to evaluate the reliability, validity, and responsiveness of the VEINES-QOL/Sym in patients with acute symptomatic DVT. We also performed an exploratory factor analysis.

Results Overall, 352 French- and German-speaking patients were enrolled (response rate of $87 \%$ ). Both language versions of the VEINES-QOL/Sym showed good acceptability (missing data, floor and ceiling effects), reliability (internal consistency, item-total and inter-item correlations), validity (convergent, discriminant, known-groups differences), and
\end{abstract}

Electronic supplementary material The online version of this article (doi:10.1007/s11136-014-0704-x) contains supplementary material, which is available to authorized users.

M. Méan $(\bowtie) \cdot$ D. Aujesky

Division of General Internal Medicine, Department of General Internal Medicine, Bern University Hospital, Inselspital,

3010 Bern, Switzerland

e-mail: marie.mean@insel.ch

A. Limacher

CTU Bern, Department of Clinical Research, and Institute of Social and Preventive Medicine (ISPM), University of Bern,

Bern, Switzerland

S. R. Kahn

Centre for Clinical Epidemiology, Jewish General Hospital,

Montreal, QC, Canada responsiveness to clinical change over time in elderly patients with DVT. The exploratory factor analysis of the VEINESQOL/Sym suggested three underlying dimensions: limitations in daily activities, DVT-related symptoms, and psychological impact.

Conclusions The VEINES-QOL/Sym questionnaire is a practical, reliable, valid, and responsive instrument to measure quality of life and symptoms in elderly patients with DVT and can be used with confidence in prospective studies to measure outcomes in such patients.

Keywords Health-related quality of life - Elderly patients - Acute deep vein thrombosis - Disease-specific quality of life questionnaire

\section{Introduction}

Acute deep vein thrombosis (DVT) is common and has a high impact on morbidity, mortality, costs of care, and quality of life $[1,2]$. Besides the transient discomfort related to acute DVT, health-related quality of life (HRQoL) is substantially influenced by the development of DVT-related complications, such as the post-thrombotic syndrome (PTS) [3].

Although the incidence rate of DVT increases markedly with advancing age [4], no study has evaluated HRQoL in elderly patients with DVT. In fact, few studies reported on HRQoL after DVT and most of them were limited by retrospective design [5] and the absence of a disease-specific HRQoL measure [6], or enrolled younger patients with DVT [2]. Given the reduced life expectancy and the observation that baseline HRQoL tends to be lower in older patients [7], HRQoL aspects may be particularly relevant in the elderly with DVT. 
Assessment of HRQoL should ideally include both a generic and a disease-specific instrument allowing outcome evaluation of patients with the same disease. The Venous Insufficiency Epidemiological and Economic Study (VEINES-QOL/Sym) questionnaire, developed to assess HRQoL in patients with venous disease, was shown to be acceptable, reliable, valid, and responsive for use in patients with DVT [8, 9]. However, given that the VEINES-QOL/Sym was developed and validated in younger patients only (mean age 46-56 years) [8, 9], we aimed to prospectively evaluate the psychometric properties of the VEINES-QOL/Sym questionnaire in a Swiss cohort of elderly patients with DVT. Our second objective was to prospectively validate the psychometric properties of a German version of the VEINES-QOL/Sym questionnaire.

\section{Methods}

\section{VEINES-QOL/Sym questionnaire}

The VEINES-QOL/Sym questionnaire is a 26-item questionnaire designed for self-completion [8]. It measures the impact of DVT on symptoms and HRQoL from the patient's perspective. The questionnaire covers DVT-related symptoms (10 items), limitations in daily activities (9 items), psychological impact (5 items), the amount of change in the respondent's leg problem over the past year (1 item), and the time of day that the leg problem is most intense (1 item). As in the widely known Short-Form Health Survey (SF-36), the time frame for questions about symptoms, limitations in daily activities, and psychological impact covers the previous 4 weeks [10]. Responses are rated on a Likert response scale.

To date, there exist English, French, Norwegian, Dutch, Turkish, and Italian language versions of the VEINESQOL/Sym questionnaire [9, 11-13], but only the English, French, and Norwegian versions were validated for use in patients with acute DVT [8, 11]. Because no German version of this questionnaire was available, we performed a forward and backward translation from the original English version of the VEINES-QOL/Sym questionnaire into German according to previously published methods [14]. In a first step, two independent native German speakers, of whom one was a translator with no medical background, performed a forward translation from the original English version into German. In a second step, two naïve English speakers performed a backward translation into English. A committee of three experts reviewed all translations and reached a consensus on any discrepancy. The final German version is shown in the supplemental online appendix.
Scoring the VEINES-QOL/Sym

Of the 26 items in the questionnaire, 25 are summed to create the VEINES-QOL summary score, which provides an estimate of the respondent's HRQoL related to the overall impact of DVT on patient's quality of life [8,9]. A subset of 10 items is summed to create a separate summary score measuring symptom severity, the VEINES-Sym score. It includes nine venous symptoms (heavy legs, aching legs, swelling, night cramps, heat or burning sensation, restless legs, throbbing, itching, and tingling sensation), rated on a 5-point scale of frequency, and leg pain, rated on a 6-point scale of intensity. The scales of items 3, 6 , and 7 are reversed, a higher score indicating a better outcomes. One item (question 2), 'At what time of day is your leg problem most intense,' provides only descriptive information and is not scored. Item $4 \mathrm{a}$ was considered missing if the answer was 'I do not work.' For both the VEINES-QOL and VEINES-Sym scales, higher scores indicate better outcomes [8,9].

As described in the initial VEINES-QOL/Sym publications [8, 9], we used the standard method for scoring questionnaires comprising items with different response scales [15]. Raw scores were first transformed to $z$-scores (mean 0; standard deviation 1) and then were averaged to form summary scores, in case where the patient answered at least $50 \%$ of the items in the summary scores. Finally, the mean $z$-scores were transformed to $T$-scores (mean 50; standard deviation 10).

Validation of the VEINES-QOL/Sym in the Swiss cohort of elderly patients with venous thromboembolism (SWITCO65+)

SWITCO65+ is an ongoing prospective multicenter cohort study to assess long-term medical outcomes and HRQoL in consecutive inpatient and outpatient aged $\geq 65$ years with acute venous thromboembolism (VTE) from all five university and four high-volume nonuniversity hospitals in Switzerland [16]. For this project, we only considered patients with objectively confirmed, acute symptomatic DVT. Symptomatic DVT was defined as an acute onset of leg pain or swelling plus incomplete compressibility of a venous segment on ultrasonography or an intraluminal filling defect on contrast venography. A detailed description of the study methods, including the detailed definition of DVT and inclusion and exclusion criteria, was previously published [16]. The study was approved by the Institutional Review Board at each participating center. 


\section{Data collection}

For all enrolled patients, trained study nurses prospectively collected baseline demographic information, comorbid conditions, laboratory findings, and VTE-related treatment, using standardized data collection forms. Follow-up included face-to-face evaluations at 3, 12, and 24 months. At each visit, patient-reported symptoms and HRQoL were assessed using the appropriate language versions (i.e., French or German) of the VEINES-QOL/Sym and SF-36 questionnaires. If patients were unable to self-complete the questionnaires, they were assisted by the study nurse. The decision to assist was left to the study nurse's discretion.

The SF-36 questionnaire is a generic HRQoL measure containing eight scales (physical functioning, social functioning, physical role functioning, emotional role functioning, mental health, vitality, bodily pain, and general health), scoring $0-100$, with higher values indicating better health $[10,17]$. Combining scales into a physical health summary (PCS) score and mental health summary (MCS) score creates two summary scores. Standardized dimension and summary scores of the SF-36 questionnaire were calculated using the US 1998 reference population [18].

At each visit, symptoms and signs of the PTS were assessed by a study nurse using a validated clinical scale developed by Villalta et al. [19]. This scale grades the severity, from 0 (absent) to 3 (severe), of each of five symptoms (pain, cramps, heaviness, pruritus, and paresthesia) and six signs (edema, skin induration, hyperpigmentation, venous ectasia, redness, and pain during calf compression). The maximum score is 33 . A score of $\geq 5$ or the presence of a venous ulcer is indicative of the PTS. The Villalta scale classifies patients with PTS into three severity categories: mild (5-9 score points), moderate (9-14 score points), and severe (>14 score points or presence of a venous ulcer).

\section{Psychometric evaluation of the VEINES-QOL/Sym questionnaire}

We used standard psychometric tests and criteria to evaluate the acceptability, reliability, validity, and responsiveness of the VEINES-QOL/Sym using questionnaires filled in at 3 months after the acute DVT because the time frame for questions in the HRQoL measures covers the preceding 4 weeks $[8,9,20]$. Hence, scores at baseline (i.e., at the time of presentation with acute DVT) would reflect a composite of pre-DVT-related and acute DVT-related HRQoL.

The study sample was divided into two groups according to the language version of the VEINES-QOL/Sym questionnaire, French and German. Baseline characteristics were shown as number and percentage or median and interquartile range (IQR), as appropriate. The transformed VEINES-QOL/Sym summary scores were depicted in a box plot as medians with interquartile ranges (IQRs) and whiskers with maximum length of $1.5 \mathrm{IQRs}$. Associations between patient characteristics and one or more missing items per patient were assessed by the chi-squared test and Wilcoxon rank-sum test as appropriate.

Acceptability affects the quality of the data obtained and was assessed by examining completeness of data and score distribution. To assess floor and ceiling effects, the proportion of patients achieving the lowest or highest possible score per summary score was calculated. Criteria for a good acceptability included $<5 \%$ missing data and $<15 \%$ floor and ceiling effects for summary scores [20].

We assessed reliability by measuring internal consistency, which was measured by Cronbach's alpha, average inter-item correlation, and item-total correlation. Internal consistency refers to the extent to which items comprising the score measure the same construct (i.e., homogeneity of the score) and was considered acceptable when Cronbach's alpha was $>0.7$. We regarded an item-total correlation $>0.2$ and an average inter-item correlation $>0.3$ as good [20].

Factor analysis is widely used to evaluate whether questionnaire items can be grouped into clusters representing different dimensions of the construct under study [21]. We performed an exploratory factor analysis with orthogonal varimax rotation on the items of both language versions of the VEINES-QOL/Sym questionnaires. The number of retained factors was determined by a scree test. Question 4a ('Does your leg problem now limit you in daily activities at work?') was omitted from the factor analysis because most patients in our sample did not work. An item was considered to load on a given factor if the loading was $>0.3$ for this factor.

Construct validity refers to the extent to which VEINESQOL/Sym summary scores relate to other measures in a manner consistent with theoretically derived hypotheses [20,22]. We first evaluated construct validity by calculating pairwise Spearman's correlations between VEINES-QOL/ Sym and SF-36 summary scores (convergent validity). Because both questionnaires assess HRQoL, we assumed that the two measures would be correlated in a moderate range, one being a disease-specific and the other a generic HRQoL questionnaire. We then evaluated correlations between VEINES-QOL/Sym summary scores and clinical characteristics (discriminant validity). Our hypothesis was that correlation with age, gender, and the presence of a concomitant comorbid condition would be low. Knowngroups differences validity was assessed by presenting the median and IQR of the VEINES summary scores by severity of the PTS based on the Villalta scale. We expected that the VEINES-QOL/Sym scores (i.e., quality of life) would decrease with increasing severity of the PTS. 
Fig. 1 Flowchart. DVT deep vein thrombosis, $H R Q o L$ health-related quality of life
446 Patients with DVT enrolled

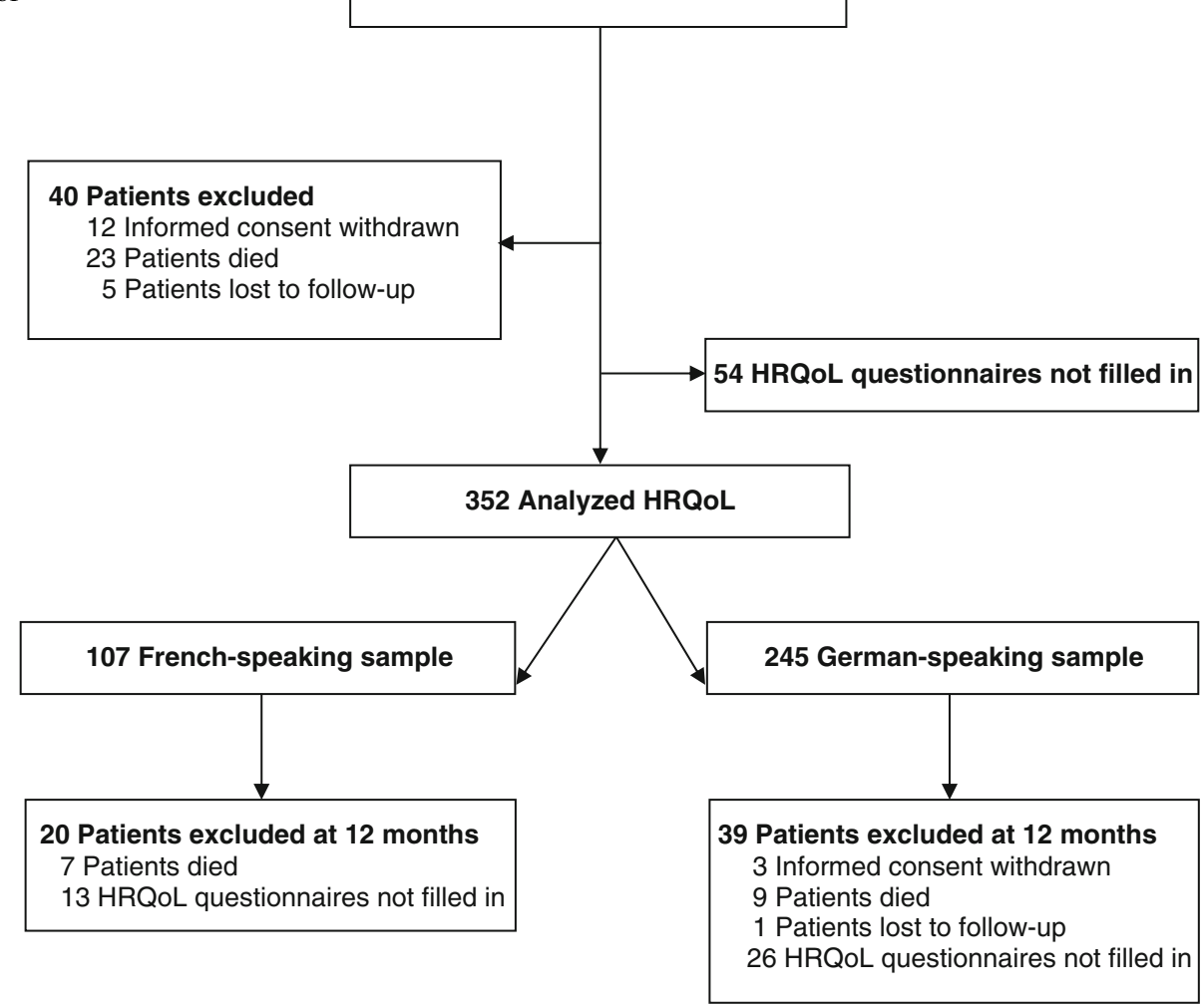

To evaluate the responsiveness of the VEINES-QOL/ Sym questionnaire, we tested the ability of the VEINESQOL/Sym to detect clinically important change over time by comparing mean changes in VEINES-QOL/Sym scores between three and 12 months after the acute DVT in patients with and without clinical improvement. Clinical improvement was defined as a decrease in DVT-related pain and severity of PTS based on the Villalta scale.

To validate the German version of the VEINES-QOL/ Sym questionnaire, we compared its psychometric properties with those of the French version, which has already been shown to be acceptable, reliable, valid, and responsive in patients with DVT $[8,9]$.

\section{Results}

Study sample

From September 2009 to March 2012, 446 patients with acute DVT were enrolled in SWITCO65+ (Fig. 1). After 3 months of follow-up, 23 patients were dead, 12 had withdrawn informed consent, and five were lost to followup. Of the 406 remaining patients, 54 did not fill in the questionnaires, leaving a final study sample of 352 responders (response rate $87 \%$ ). Responders had a median age of 74 years, 161 (46\%) were female, $269(76 \%)$ were not working, and $57(16 \%)$ had an active cancer. Overall, $107(30 \%)$ were French speakers, and 245 (70\%) were German speakers. French speakers were somewhat older and had more comorbid illnesses than German-speaking patients (Table 1). Non-responders were older than responders (median age 78 vs. 74 years; $P=0.02$ ) and were more likely to have a concomitant overt pulmonary embolism (54 vs. $27 \%$; $P<0.001)$.

Psychometric properties of the VEINES-QOL/Sym

The median-transformed VEINES-QOL and VEINES-Sym summary scores were 48.5 (IQR 36.8-56.6) and 50.4 (IQR 40.2-56.8) in the French-speaking sample and 54.4 (IQR 46.2-59.1) and 53.9 (IQR 47.4-58.7) in the Germanspeaking sample, respectively.

\section{Acceptability}

Both language versions showed good acceptability, with low proportions of missing data and floor/ceiling effects (Table 2). Overall, $12 \%$ of patients had $\geq 1$ missing items. Missing items ranged between 2 and $9 \%$ in the Frenchspeaking sample and 0 and $1 \%$ in the German-speaking sample. French speakers were more likely to have one or 
Table 1 Patient characteristics

\begin{tabular}{llc}
\hline & $\begin{array}{l}\text { French- } \\
\text { speaking } \\
\text { sample } \\
(N=107)\end{array}$ & $\begin{array}{l}\text { German- } \\
\text { speaking } \\
\text { sample } \\
(N=245)\end{array}$ \\
& Median (IQR) & or $(\%)$ \\
\hline Age (years) & $76(68-80)$ & $74(69-80)$ \\
Female gender & $53(50)$ & $108(44)$ \\
Less than high school as highest & $55(51)$ & $133(54)$ \\
educational level & & \\
Not working & $77(72)$ & $192(78)$ \\
Living in nursing home & $0(0)$ & $6(2)$ \\
Prior venous thromboembolism & $41(38)$ & $73(30)$ \\
Outpatient & $52(49)$ & $94(38)$ \\
Overt pulmonary embolism & $28(26)$ & $68(28)$ \\
Active cancer & $22(21)$ & $35(14)$ \\
Cardiac disease & $19(18)$ & $48(20)$ \\
Chronic lung disease & $12(11)$ & $24(10)$ \\
Cerebrovascular disease & $4(4)$ & $19(8)$ \\
Diabetes mellitus & $22(21)$ & $34(14)$ \\
Villalta score & $5(3-8)$ & $4(2-6)$ \\
\hline
\end{tabular}

$I Q R$ interquartile range

${ }^{a}$ At 3 months after the index venous thromboembolic event

more missing items than German speakers (28 vs. $4 \%$; $P<0.001)$, and women had more missing items than men (17 vs. $7 \% ; P=0.006$ ). Age was not associated with missingness.

\section{Factor analysis}

The factor analyses suggested three underlying latent factors (dimensions) for both questionnaires: limitations in daily activities (items 4b-d, 5a-d, 6, and 7), DVT-related symptoms (items 1a-i), and psychological impact (items 8a-e). Item 3 ('Compared to 1 year ago, how would you rate your leg problem in general now?') did not load on any factor (loadings $\leq 0.3$ ), indicating that it could be omitted from the VEINES-QOL summary score. The detailed factor analyses are shown in the supplemental online appendix.

\section{Internal consistency reliability}

Cronbach's alpha coefficients were $>0.8$ in both language versions, indicating high internal consistency (Table 2) [20]. Items were positively correlated with each other, with average inter-item correlations of $>0.3$ (Table 2). Almost all item-total correlation values were $>0.2$, ranging from 0.42 to 0.79 for the French-speaking sample and from 0.35 to 0.80 for the German-speaking sample [20]. Only item 3, asking: 'Compared to 1 year ago, how would you rate your
Table 2 Acceptability and internal consistency reliability of the VEINES-QOL/Sym

\begin{tabular}{lll}
\hline & $\begin{array}{l}\text { French-speaking } \\
\text { sample } \\
(N=107)\end{array}$ & $\begin{array}{l}\text { German-speaking } \\
\text { sample } \\
(N=245)\end{array}$ \\
\hline $\begin{array}{l}\text { Scorable questionnaires } \\
\text { VEINES-QOL }\end{array}$ & 100 & 100 \\
VEINES-Sym & 96 & 100 \\
Floor effects $(\%)$ & & \\
VEINES-QOL & 0 & 0 \\
VEINES-Sym & 0 & 0 \\
Ceiling effects $(\%)$ & & \\
VEINES-QOL & 0 & 0.4 \\
VEINES-Sym & 7.8 & 12.2 \\
Cronbach's alpha & & \\
VEINES-QOL & 0.93 & 0.92 \\
VEINES-Sym & 0.88 & 0.82 \\
Average inter-item correlation, $r$ & \\
VEINES-QOL & 0.34 & 0.31 \\
VEINES-Sym & 0.43 & 0.32 \\
\hline
\end{tabular}

${ }^{a}$ Defined as $50 \%$ or more of items completed

leg problem in general now?', was not correlated with other items (item-total correlation of 0.18 and -0.10 , respectively), suggesting again that this item could be omitted from the VEINES-QOL summary score. Ceiling and floor statistics, Cronbach's alpha, and item-score correlations for each individual item are shown in the supplemental online appendix.

Construct validity (convergent, discriminant, and knowngroups differences validity)

We did a correlation analysis using SF-36 component scores to assess convergent validity of the VEINES-QOL/ Sym summary scores (Table 3). In both language versions, the VEINES-QOL/Sym was moderately correlated with SF-36 summary scores and showed higher correlations for the PCS than for the MCS. In general, the VEINES-QOL summary scores showed a better correlation with the SF-36 summary scores than the VEINES/Sym summary scores. Age, gender, and the presence of a concomitant comorbid condition were very weakly correlated with VEINES-QOL and VEINES/Sym summary scores $(r<0.19)$ in both language versions, indicating a good discriminant validity. This suggests that responses to the VEINES-QOL/Sym are not biased in terms of age, gender, or a concomitant comorbid condition (Table 3 ).

In both language versions, the VEINES-QOL/Sym summary scores demonstrated lower scores (i.e., poorer QOL) with increasing severity of PTS, demonstrating that 
Table 3 Convergent and discriminant validity of the VEINES-QOL/ Sym

\begin{tabular}{ccclll}
\hline & \multicolumn{2}{l}{$\begin{array}{l}\text { French-speaking sample } \\
(N=107)\end{array}$} & & \multicolumn{2}{l}{$\begin{array}{l}\text { German-speaking } \\
\text { sample }(N=245)\end{array}$} \\
\cline { 2 - 3 } & $\begin{array}{l}\text { VEINES- } \\
\text { QOL }\end{array}$ & $\begin{array}{l}\text { VEINES- } \\
\text { Sym }\end{array}$ & & $\begin{array}{l}\text { VEINES- } \\
\text { QOL }\end{array}$ & $\begin{array}{l}\text { VEINES- } \\
\text { Sym }\end{array}$ \\
\hline SF-36 & & & & \\
PCS & $0.72^{*}$ & $0.52^{*}$ & & $0.62^{*}$ & $0.45^{*}$ \\
MCS & $0.66^{*}$ & $0.51^{*}$ & & $0.44^{*}$ & $0.32^{*}$ \\
Patient characteristics & & & \\
Age & -0.04 & 0.07 & $-0.14^{\dagger}$ & $-0.14^{\dagger}$ \\
Female & -0.16 & -0.12 & $-0.19^{\dagger}$ & $-0.14^{\dagger}$ \\
gender & & & & -0.02 \\
Comorbidity & -0.05 & -0.04 & -0.07 & -0.02 \\
Active cancer & -0.11 & -0.01 & -0.09 & -0.02
\end{tabular}

PCS physical health summary score, $M C S$ mental health summary score

$* P<0.001$

${ }^{\dagger} P<0.05$

* Presence of cardiac, cerebrovascular, or chronic lung disease, or diabetes mellitus

known-groups differences validity was good (Table 4). In summary, all correlations supported our hypotheses and confirmed the construct validity of the VEINES-QOL/Sym questionnaire in elderly patients with DVT.

\section{Responsiveness}

After 12 months, 293 out of $332(88 \%)$ patients who attended the follow-up visit completed the questionnaires and were available for our responsiveness analysis. In this analysis, we compared the summary score changes of the VEINES-QOL/Sym questionnaire between three and 12 months after the acute DVT in patients with and without a clinical improvement with respect to DVT-related pain and severity of the PTS (Table 5). In the German questionnaire, the VEINES-QOL and VEINES/Sym summary scores were both significantly responsive to clinical change. Differences in mean change scores between patients with clinical improvement and patients without clinical improvement ranged between 4.9 and 2.2 points ( $P$ values $\leq 0.04$ for all comparisons).

In the French questionnaire, we measured a larger change in VEINES-QOL and VEINES/Sym scores in patients who had clinically improved. Differences in mean change scores between patients with clinical improvement and patients without clinical improvement ranged between 4.9 and 0.7 points. However, the difference in change only achieved statistical significance for the VEINES-QOL score when comparing patients with and without improvement of DVTrelated pain $(P=0.02)$.

\section{Discussion}

Our results demonstrate that both the French and German versions of the VEINES-QOL/Sym questionnaire meet standard criteria of acceptability, reliability, validity, and responsiveness for use as a patient-reported measure of outcome in elderly patients with acute DVT. A number of other disease-specific quality of life instruments intended for use in patients with DVT have been proposed, but none has been validated in elderly patients [23-25]. Thus, although the VEINES-QOL/Sym was developed and validated in younger patients (mean age 46-56 years) $[8,9,11]$, it can also be applied with confidence to elderly patients.

In this sample of elderly patients with acute DVT, the response rate was $87 \% 3$ months after the acute DVT event. Our response rate compares well to studies enrolling younger patients in whom the response rates for the VEINES-QOL/Sym ranged from 72 to $100 \%$ [8, 11, 12]. In our study, although assistance was offered to patients who

Table 4 Known-groups differences validity of the VEINES-QOL/Sym*

\begin{tabular}{|c|c|c|c|c|c|c|}
\hline & \multicolumn{3}{|c|}{ French-speaking sample $(N=107)$} & \multicolumn{3}{|c|}{ German-speaking sample $(N=245)$} \\
\hline & $N$ & $\begin{array}{l}\text { VEINES-QOL score, } \\
\text { median (IQR) }\end{array}$ & $\begin{array}{l}\text { VEINES-Sym score, } \\
\text { median (IQR) }\end{array}$ & $N$ & $\begin{array}{l}\text { VEINES-QOL score, } \\
\text { median (IQR) }\end{array}$ & $\begin{array}{l}\text { VEINES-Sym score, } \\
\text { median (IQR) }\end{array}$ \\
\hline Overall & 107 & $48.5(36.8-56.6)$ & $50.4(40.2-56.8)$ & 232 & $54.3(46.2-59.1)$ & $53.9(47.4-58.8)$ \\
\hline No PTS & 47 & $50.1(42.0-57.5)$ & $52.2(45.2-58.7)$ & 130 & $57.2(50.2-60.5)$ & $57.4(51.5-60.5)$ \\
\hline Mild PTS & 43 & $48.7(36.0-56.6)$ & $50.4(41.8-57.5)$ & 77 & $52.3(45.4-57.1)$ & $50.8(45.0-54.6)$ \\
\hline Moderate PTS & 8 & $36.3(28.7-52.4)$ & $37.9(32.0-55.0)$ & 16 & $39.0(33.3-50.7)$ & $42.4(30.2-49.8)$ \\
\hline Severe PTS & 9 & $36.5(30.8-43.3)$ & $37.3(27.4-47.4)$ & 9 & $37.7(36.3-38.9)$ & $39.1(36.3-45.8)$ \\
\hline$P$ value ${ }^{\dagger}$ & - & 0.003 & 0.02 & - & $<0.001$ & $<0.001$ \\
\hline
\end{tabular}

*Expressed as median VEINES-QOL/Sym scores by severity of the PTS based on the Villalta scale [19]

$I Q R$ interquartile range, PTS post-thrombotic syndrome

${ }^{\dagger} P$ value for difference between PTS-groups based on a Kruskal-Wallis test 
Table 5 Responsiveness of the VEINES-QOL/Sym

\begin{tabular}{|c|c|c|c|c|c|c|}
\hline & \multicolumn{3}{|c|}{ French-speaking sample $(N=87)$} & \multicolumn{3}{|c|}{ German-speaking sample $(N=206)$} \\
\hline & $\begin{array}{l}\text { Clinically* } \\
\text { improved } \\
\text { Mean change } \\
\text { scores }^{\dagger}(n)^{*}\end{array}$ & $\begin{array}{l}\text { Clinically* } \\
\text { unimproved }\end{array}$ & $P$ value $^{\S}$ & $\begin{array}{l}\text { Clinically* } \\
\text { improved } \\
\text { Mean change } \\
\text { scores }^{\dagger}(n)^{*}\end{array}$ & $\begin{array}{l}\text { Clinically* } \\
\text { unimproved }\end{array}$ & $P$ value $^{\S}$ \\
\hline \multicolumn{7}{|c|}{ Deep vein thrombosis-related pain } \\
\hline VEINES-QOL & $4.9(10)$ & $0.0(77)$ & 0.02 & $3.4(32)$ & $0.2(171)$ & 0.02 \\
\hline VEINES-Sym & $3.5(9)$ & $0.3(74)$ & 0.17 & $4.2(32)$ & $-0.7(171)$ & 0.002 \\
\hline \multicolumn{7}{|c|}{ Severity of post-thrombotic syndrome } \\
\hline VEINES-QOL & $1.0(38)$ & $0.3(49)$ & 0.59 & $2.1(89)$ & $-0.1(108)$ & 0.03 \\
\hline VEINES-Sym & $1.3(37)$ & $0.0(46)$ & 0.37 & $1.4(89)$ & $-1.0(108)$ & 0.04 \\
\hline
\end{tabular}

were unable to self-complete the questionnaires, $13 \%$ of patients who attended the follow-up visit at 3 months did not complete the questionnaires. The reason may be that non-responders were significantly older and sicker than responders. Previous studies validating the use of the SF-36 in older adults found that non-completion was associated with increasing age and that persons with poor physical and mental health scores felt more often unable to self-complete the SF-36 and required assistance [7, 26]. The fact that patients were required to complete a total of three HRQoL questionnaires (SF-36, VEINES-QOL/Sym, and a pulmonary-embolism-related HRQoL questionnaire) in SWITCO65+ may also have reduced the response rate. A high questionnaire burden was previously shown to be an obstacle to study participation and adherence in elderly patients and should be minimized when designing studies focusing on the elderly [27].

In our sample of elderly patients with DVT, the exploratory factor analysis supported the formation of three dimensions [limitations in daily activities, (items 4, 5, 6, and 7); DVT-related symptoms, (item 1); and psychological impact, (item 8)]. Question 3 ('Compared to 1 year ago, how would you rate your leg problem in general now?') did not load on any factor and was not correlated with other items, indicating that it could be omitted without loss of information in elderly patients with acute DVT. This finding is consistent with the results from a previous validation study of patients with chronic venous disease, in which question 3 did not sufficiently load on the factor in a confirmatory factor analysis [12]. Moreover, when answering question 4a ('Does your leg problem now limit you in daily activities at work'), three quarter of our patients responded 'I do not work', presumably, because the majority of persons aged over 65 years are retired. This suggests that this question may not be relevant and could be omitted in elderly patients. Finally, to shorten the time required to complete the VEINES-QOL/Sym, question 2 ('At what time of day is your leg problem most intense') could also be omitted because it provides only descriptive information and is not scored. The omission of these three questions has the potential to make the VEINES-QOL/Sym questionnaire more user friendly for elderly patients and may further increase the response rate. However, the validation of such a VEINES-QOL/Sym version for the elderly is required before its use can be recommended.

Compared to German-speaking patients, French-speaking patients were somewhat older and more likely to suffer from prior venous thromboembolism, active cancer, and diabetes mellitus, which could explain the lower median VEINES-QOL/Sym scores and higher rate of missing items in the French-speaking sample.

Our study has potential limitations. First, we were not able to assess test-retest reliability of the VEINES-QOL/ Sym, which is an indicator of the stability of a measuring instrument. Previous studies enrolling younger patients found a good test-retest reliability (intraclass correlation coefficients of $>0.8$ ) for the VEINES-QOL/Sym in patients with DVT [8, 11]. Second, the German version of the VEINES-QOL/Sym questionnaire met standard criteria of acceptability, reliability, validity, and responsiveness for use in elderly patients with acute DVT, but it needs to be validated in younger patients before its use can be recommended in such patients. Third, this sample may not reflect the full prognostic spectrum of patients with acute DVT because responders were younger and healthier than non-responders. Thus, we cannot exclude the possibility 
that our results would differ in more severe ill patients. Finally, we did not ascertain the proportion of patients who needed assistance to complete the questionnaires. Thus, we would expect a higher proportion of non-responders and missing items if the questionnaires were based on selfcompletion only.

In conclusion, our results demonstrate that the VEINESQOL/Sym is a practical, reliable, valid, and responsive instrument to measure DVT-specific HRQoL in elderly patients. Thus, disease-specific quality of life and symptom assessment based on the VEINES-QOL/Sym can be incorporated with confidence in prospective studies of elderly patients with acute DVT.

Acknowledgments This study was supported by Swiss National Science Foundation Grant 33CSCO-122659/139470. We thank all the investigators and the study personnel who made the SWITCO65+ study possible (in particular, Marc Righini, Kurt Jaeger, Hans-Jürg Beer, Beat Frauchiger, Joseph Osterwalder, Nils Kucher, Bernhard Lämmle, Jacques Cornuz, Anne Angelillo-Scherrer, Nicolas Rodondi, Sven Trelle, Christian M. Matter, Marc Husmann, Martin Banyai, Markus Aschwanden, Michael Egloff, Lucia Mazzolai, Olivier Hugli, and Henri Bounameaux).

Conflict of interest The authors have no conflict of interest.

\section{References}

1. Cohen, A. T., Agnelli, G., Anderson, F. A., Arcelus, J. I., Bergqvist, D., Brecht, J. G., et al. (2007). Venous thromboembolism (VTE) in Europe. The number of VTE events and associated morbidity and mortality. Thrombosis and Haemostasis, 98(4), 756-764.

2. Kahn, S. R., Ducruet, T., Lamping, D. L., Arsenault, L., Miron, M. J., Roussin, A., et al. (2005). Prospective evaluation of healthrelated quality of life in patients with deep venous thrombosis. Archives of Internal Medicine, 165(10), 1173-1178.

3. Kahn, S. R., \& Ginsberg, J. S. (2004). Relationship between deep venous thrombosis and the postthrombotic syndrome. Archives of Internal Medicine, 164(1), 17-26.

4. Spencer, F. A., Gore, J. M., Lessard, D., Emery, C., Pacifico, L., Reed, G., et al. (2008). Venous thromboembolism in the elderly. A community-based perspective. Thrombosis and Haemostasis, 100(5), 780-788.

5. Beyth, R. J., Cohen, A. M., \& Landefeld, C. S. (1995). Long-term outcomes of deep-vein thrombosis. Archives of Internal Medicine, 155(10), 1031-1037.

6. Koopman, M. M., Prandoni, P., Piovella, F., Ockelford, P. A., Brandjes, D. P., van der Meer, J., et al. (1996). Treatment of venous thrombosis with intravenous unfractionated heparin administered in the hospital as compared with subcutaneous lowmolecular-weight heparin administered at home. The Tasman Study Group. New England Journal of Medicine, 334(11), 682-687.

7. Walters, S. J., Munro, J. F., \& Brazier, J. E. (2001). Using the SF36 with older adults: A cross-sectional community-based survey. Age and Ageing, 30(4), 337-343.

8. Kahn, S. R., Lamping, D. L., Ducruet, T., Arsenault, L., Miron, M. J., Roussin, A., et al. (2006). VEINES-QOL/Sym questionnaire was a reliable and valid disease-specific quality of life measure for deep venous thrombosis. Journal of Clinical Epidemiology, 59(10), 1049-1056.

9. Lamping, D. L., Schroter, S., Kurz, X., Kahn, S. R., \& Abenhaim, L. (2003). Evaluation of outcomes in chronic venous disorders of the leg: Development of a scientifically rigorous, patient-reported measure of symptoms and quality of life. Journal of Vascular Surgery, 37(2), 410-419.

10. Ware, J. E., Jr, \& Sherbourne, C. D. (1992). The MOS 36-item short-form health survey (SF-36). I. Conceptual framework and item selection. Medical Care, 30(6), 473-483.

11. Enden, T., Garratt, A. M., Klow, N. E., \& Sandset, P. M. (2009). Assessing burden of illness following acute deep vein thrombosis: Data quality, reliability and validity of the Norwegian version of VEINES-QOL/Sym, a disease-specific questionnaire. Scandinavian Journal of Caring Sciences, 23(2), 369-374.

12. van der Velden, S. K., Biemans, A. A., Nijsten, T., \& Sommer, A. (2014). Translation and validation of the Dutch VEINES-QOL/ Sym in varicose vein patients. Phlebology, 29(4), 227-235. doi: $10.1177 / 0268355513476279$

13. Tuygun, A. K., Ketenci, B., Gunay, R., Gorur, A., Guney, M. R., Bicer, M., et al. (2012). Validity and reliability of VEINES-QOL/ Sym questionnaire in chronic venous disorders. The Journal of Cardiovascular Surgery (Torino), 53(3), 355-361.

14. Beaton, D. E., Bombardier, C., Guillemin, F., \& Ferraz, M. B. (2000). Guidelines for the process of cross-cultural adaptation of self-report measures. Spine (Phila Pa 1976), 25(24), 3186-3191.

15. Streiner, D. L., \& Norman, G. (1995). Health measurement scales: A practical guide to their development and use (2nd ed.). Oxford: Oxford University Press.

16. Méan, M., Righini, M., Jaeger, K., Beer, H. J., Frauchiger, B., Osterwalder, J., et al. (2013). The Swiss cohort of elderly patients with venous thromboembolism (SWITCO65+): Rationale and methodology. Journal of Thrombosis and Thrombolysis, 36(4), $475-483$.

17. McHorney, C. A., Ware, J. E., Jr, \& Raczek, A. E. (1993). The MOS 36-Item Short-Form Health Survey (SF-36): II. Psychometric and clinical tests of validity in measuring physical and mental health constructs. Medical Care, 31(3), 247-263.

18. Ware, J. E., Jr. (2000). SF-36 health survey update. Spine (Phila Pa 1976), 25(24), 3130-3139.

19. Villalta, S., Bagatella, P., Piccioli, A., Lensing, A. W., Prins, M. H., \& Prandoni, P. (1994). Assessment of validity and reproducibility of a clinical scale for the post-thrombotic syndrome [abstract]. Haemostasis, 24, 158a.

20. Terwee, C. B., Bot, S. D., de Boer, M. R., van der Windt, D. A., Knol, D. L., Dekker, J., et al. (2007). Quality criteria were proposed for measurement properties of health status questionnaires. Journal of Clinical Epidemiology, 60(1), 34-42.

21. de Vet, H. C., Ader, H. J., Terwee, C. B., \& Pouwer, F. (2005). Are factor analytical techniques used appropriately in the validation of health status questionnaires? A systematic review on the quality of factor analysis of the SF-36. Quality of Life Research, 14(5), 1203-1224.

22. Guyatt, G. H., Deyo, R. A., Charlson, M., Levine, M. N., \& Mitchell, A. (1989). Responsiveness and validity in health status measurement: A clarification. Journal of Clinical Epidemiology, 42(5), 403-408.

23. van Korlaar, I. M., Vossen, C. Y., Rosendaal, F. R., Bovill, E. G., Cushman, M., Naud, S., et al. (2004). The impact of venous thrombosis on quality of life. Thrombosis Research, 114(1), 11-18.

24. Hedner, E., Carlsson, J., Kulich, K. R., Stigendal, L., Ingelgard, A., \& Wiklund, I. (2004). An instrument for measuring healthrelated quality of life in patients with Deep Venous Thrombosis (DVT): Development and validation of Deep Venous Thrombosis Quality of Life (DVTQOL) questionnaire. Health and Quality of Life Outcomes, 2, 30. 
25. Hudgens, S. A., Cella, D., Caprini, C. A., \& Caprini, J. A. (2003). Deep vein thrombosis: Validation of a patient-reported leg symptom index. Health and Quality of Life Outcomes, 1, 76.

26. Hayes, V., Morris, J., Wolfe, C., \& Morgan, M. (1995). The SF36 health survey questionnaire: Is it suitable for use with older adults? Age and Ageing, 24(2), 120-125.
27. McMurdo, M. E., Roberts, H., Parker, S., Wyatt, N., May, H., Goodman, C., et al. (2011). Improving recruitment of older people to research through good practice. Age and Ageing, 40(6), $659-665$. 